\title{
Bibliothèques monastiques en monde syriaque : l'exemple des manuscrits de Charfet
}

\section{Françoise Briquel Chatonnet}

\section{(2) OpenEdition}

1 Journals

Édition électronique

URL : http://journals.openedition.org/rbnu/856

DOI : $10.4000 /$ rbnu. 856

ISSN : 2679-6104

Éditeur

Bibliothèque nationale et universitaire de Strasbourg

Édition imprimée

Date de publication : 1 mai 2017

Pagination : 32-39

ISBN : 9782859230661

ISSN : 2109-2761

Référence électronique

Françoise Briquel Chatonnet, «Bibliothèques monastiques en monde syriaque : l'exemple des manuscrits de Charfet », La Revue de la BNU [En ligne], 15 | 2017, mis en ligne le 01 novembre 2019, consulté le 11 décembre 2020. URL : http://journals.openedition.org/rbnu/856 ; DOI : https://doi.org/ $10.4000 /$ rbnu. 856

\section{(c) (1) ()}

La Revue de la BNU est mise à disposition selon les termes de la Licence Creative Commons Attribution - Pas d'Utilisation Commerciale - Partage dans les Mêmes Conditions 4.0 International. 


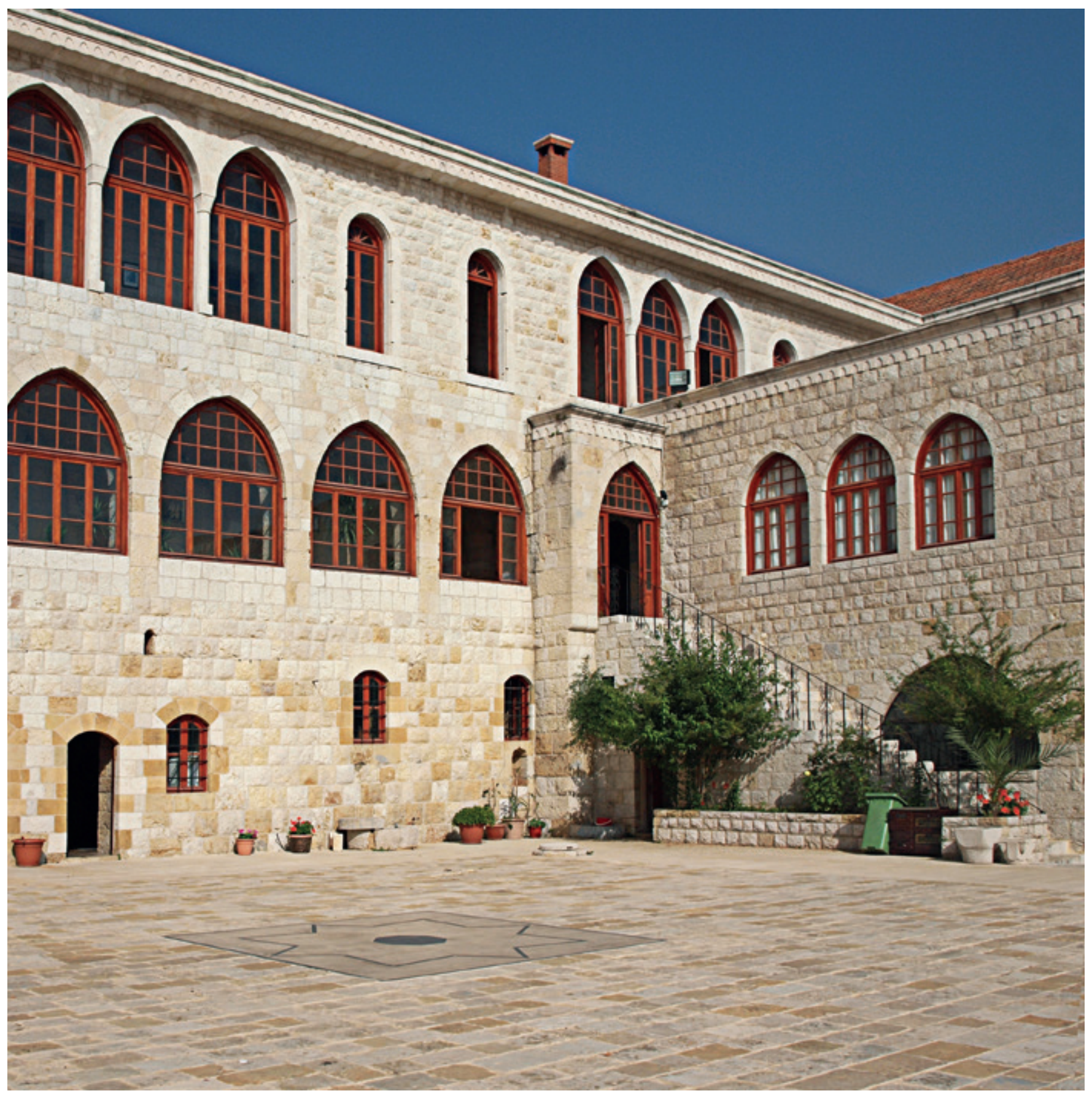

Charfet : le couvent où se trouve

la bibliothèque de manuscrits 


\section{BIBLIOTHĖQUES MONASTIQUES EN MONDE SYRIAQUE : l'exemple des manuscrits de Charfet \\ PAR FRANÇOISE BRIQUEL CHATONNET}

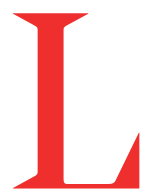

a bibliothèque du patriarcat syrocatholique, préservée dans le monastère de Charfet, au Liban (voir ill. ci-contre), est située dans la montagne au-dessus de Jounieh, dans l'ancienne résidence patriarcale qui est restée aussi le séjour d'été des patriarches. La collection comprend environ 2000 manuscrits syriaques, garshuni (arabe en caractères syriaques) et arabes. C'est donc une des plus belles collections de manuscrits syriaques du Proche-Orient, même si elle ne contient pas, à une exception près, de manuscrits très anciens.

De fait, deux collections exceptionnelles ont conservé des manuscrits syriaques anciens, celle du monastère Sainte-Catherine du Sinaï pour les manuscrits chalcédoniens, celle de Notre-Dame des Syriens (Deir as-Suriani), dans le désert de Scété en Égypte, pour les manuscrits syro-orthodoxes. Dans un cas comme dans l'autre, une partie des collections a été dispersée dans les bibliothèques européennes au 19 siècle, mais de nouvelles découvertes ont été faites au $20^{\mathrm{e}}$, dans des pièces qui avaient été fermées et scellées. Si ce sont ces deux collections qui ont conservé des manuscrits particulièrement anciens (le plus ancien manuscrit syriaque daté, provenant de Deir asSuriani, est le ms. British Library Add. 12150, de 411 après J.-C.), cela tient à la fois au climat très sec de l'Égypte, favorable à leur conservation, mais aussi et surtout au fait que ce dernier pays a été relativement à l'écart des invasions, alors que le Proche-Orient a subi des ravages et que les monastères où les manus- crits étaient conservés ont été souvent pillés et détruits. Parmi les collections proche-orientales, celle de Charfet est l'une des plus importantes (voir ill. p. 35). Les manuscrits syriaques qui la composent font l'objet depuis quelques années, à l'invitation du patriarche Mar Ignace Pierre III Abd el-Ahad et de la confiance renouvelée de son successeur, Mar Ignace Joseph II Younan, de la part de membres du CNRS appartenant aux laboratoires Orient et Méditerranée et IRHT, d'un programme de catalogage mené en collaboration avec les responsables de la bibliothèque, Mgr Gabriel Dib et Youssef Dergham ${ }^{1}$. Le premier tome du catalogue sera publié prochainement.

On appelle syriaque l'araméen utilisé par les chrétiens du Proche-Orient. C'est la langue et l'écriture d'Édesse, aujourd'hui Sanlı Urfa en Turquie, qui était au début de notre ère la capitale du petit royaume d'Osrhoène, devenu ensuite province de l'empire romain. Édesse a joué un rôle essentiel dans la christianisation de l'Orient, ce qu'évoquent différents textes de la littérature que nous qualifions d'« apocryphes " : histoire d'Abgar, roi d'Édesse, qui aurait échangé des messages avec Jésus et à qui Thomas, après l'Ascension, aurait envoyé le disciple Addaï qui convertit le roi et son royaume ; histoire de Mari, un autre des soixante-douze disciples, parti d'Édesse pour évangéliser toute la Mésopotamie jusqu'au golfe Persique ; histoire enfin de l'apôtre Thomas, lié lui aussi à Édesse et qui aurait porté l'Évangile jusqu'en Inde. Dès le $2^{\mathrm{e}}$ siècle et le début du $3^{\mathrm{e}}$, une littérature en araméen chrétien a été composée à Édesse : version 
syriaque de l'Ancien Testament (Peshitta), œuvre du philosophe Bardesane, Diatessaron (ou Évangile concordant) de Tatien, Actes de l'apôtre Thomas, etc. Avec la christianisation, la culture syriaque (chrétienne édessénienne) s'est ainsi répandue de la Méditerranée au plateau iranien, avec une expansion missionnaire très importante dans le golfe Persique jusqu'en Inde du Sud, et sur la route terrestre, en Asie centrale et jusqu'en Chine.

Ce qui est particulièrement remarquable est que cette culture n'a jamais été celle d'une structure politique. Les chrétiens de culture syriaque ont toujours vécu dans des États dont ils ne partageaient pas la religion revendiquée : le zoroastrisme était la religion officielle de l'empire sassanide ; l'empire byzantin a affiché presque continuellement son soutien à la foi définie lors du concile de Chalcédoine, qui a été rejetée par la majorité des chrétiens syriaques de l'empire, et a régulièrement persécuté les chrétiens anti-chalcédoniens ; après la conquête arabo-musulmane au $7^{\text {e }}$ siècle, les syriaques se sont trouvés sous le même pouvoir musulman. Dans leur expansion en Asie centrale, en Chine, en Inde, ils se sont toujours trouvés face à des pouvoirs non chrétiens. La culture syriaque n'a donc pas été celle d'une société civile et par conséquent, les manuscrits syriaques n'ont jamais été commandités par des élites civiles ou des membres de la cour : c'est dans le cadre des Églises qu'on les a copiés et conservés, et leur contenu est presque entièrement religieux. Chaque paroisse et chaque église devaient avoir les livres nécessaires au culte, lectionnaires de la Bible et surtout du Nouveau Testament, psautiers et livres liturgiques. Les ouvrages de culture étaient copiés et conservés dans les monastères qui ont fleuri en Syrie comme en Mésopotamie dès la fin du $4^{\mathrm{e}}$ siècle. C'est donc dans ces lieux qu'étaient formés les copistes qui maîtrisaient la belle écriture classique ( « estrangelā») et c'est pour l'usage monastique que les manuscrits ont été copiés : lecture de la Bible et méditation, mais aussi travail intellectuel. Les manuscrits comprenaient avant tout le texte de la Bible, souvent ordonné selon l'ordre des lectures, des commentaires exégétiques, des livres d'hagiographie (notamment, dans chaque monastère, la vie du fondateur et des premiers membres de la communauté locale), d'ascétisme et de mystique. Ce sont en outre des moines qui ont composé des ouvrages d'historiographie, de patristique, des homéliaires et répertoires d'hymnes, et qui ont traduit et enrichi la tradition scientifique grecque, dans des domaines comme la médecine, l'astronomie, la grammaire mais aussi la philosophie, avant de la transmettre au monde arabo-musulman.

La copie des manuscrits était une œuvre pieuse, et les copistes revendiquaient ce travail pour s'acquérir des mérites auprès de Dieu. Les colophons précisent bien sûr le nom du copiste et souvent celui du commanditaire ou du mécène qui a payé les matériaux. Mais il est bien noté que le copiste ne s'est pas fait payer, qu'il l'a fait pour le pardon de ses péchés et qu'il demande aux lecteurs de prier pour lui. C'est seulement au $19^{\mathrm{e}}$ siècle qu'apparaissent des copistes laïcs, parfois des familles de copistes, car on a continué à copier à la main des manuscrits jusqu'au début du $20^{\mathrm{e}}$ siècle, notamment en Mésopotamie.

La culture syriaque est celle de plusieurs Églises différentes, correspondant à deux grandes traditions. La culture syriaque orientale s'est formée dans l'Antiquité au sein de l'empire sassanide, en Mésopotamie et sur le plateau iranien. Elle s'est cristallisée dans une Église autocéphale, l'Église apostolique de l'Orient, d'abord pour des raisons géostratégiques : après la conversion de l'empire romain, les chrétiens de l'empire perse avec lequel l'empire sassanide était souvent en guerre ont tenu à affirmer qu'ils ne dépendaient pas d'une instance romaine. Ce n'est que dans un second temps qu'ils ont refusé de reconnaître le concile d'Éphèse, auquel ils n'avaient d'ailleurs pas participé. Cette Église a été qualifiée par ses détracteurs de "nestorienne ", mais ne s'est jamais reconnue dans cette dénomination et depuis la fin du $19^{\text {e }}$ siècle, elle est parfois appelée " assyrienne ». Elle a depuis le $16^{\mathrm{e}}$ siècle une Église-sœur catholique, l'Église chaldéenne. L'autre tradition, syro-occidentale, est née dans le monde romain et byzantin, mais elle a connu aussi une expansion vers la Mésopotamie dès la fin de l'Antiquité, à la fois grâce à un processus missionnaire, mais aussi à la suite de déportations opérées par les souverains sassanides lors d'invasions : elle est partagée entre les syro-orthodoxes, les syrocatholiques et les maronites. Ces deux traditions, syroorientale et syro-occidentale, partagent la même Bible, des œuvres comme celles du grand poète Éphrem de 


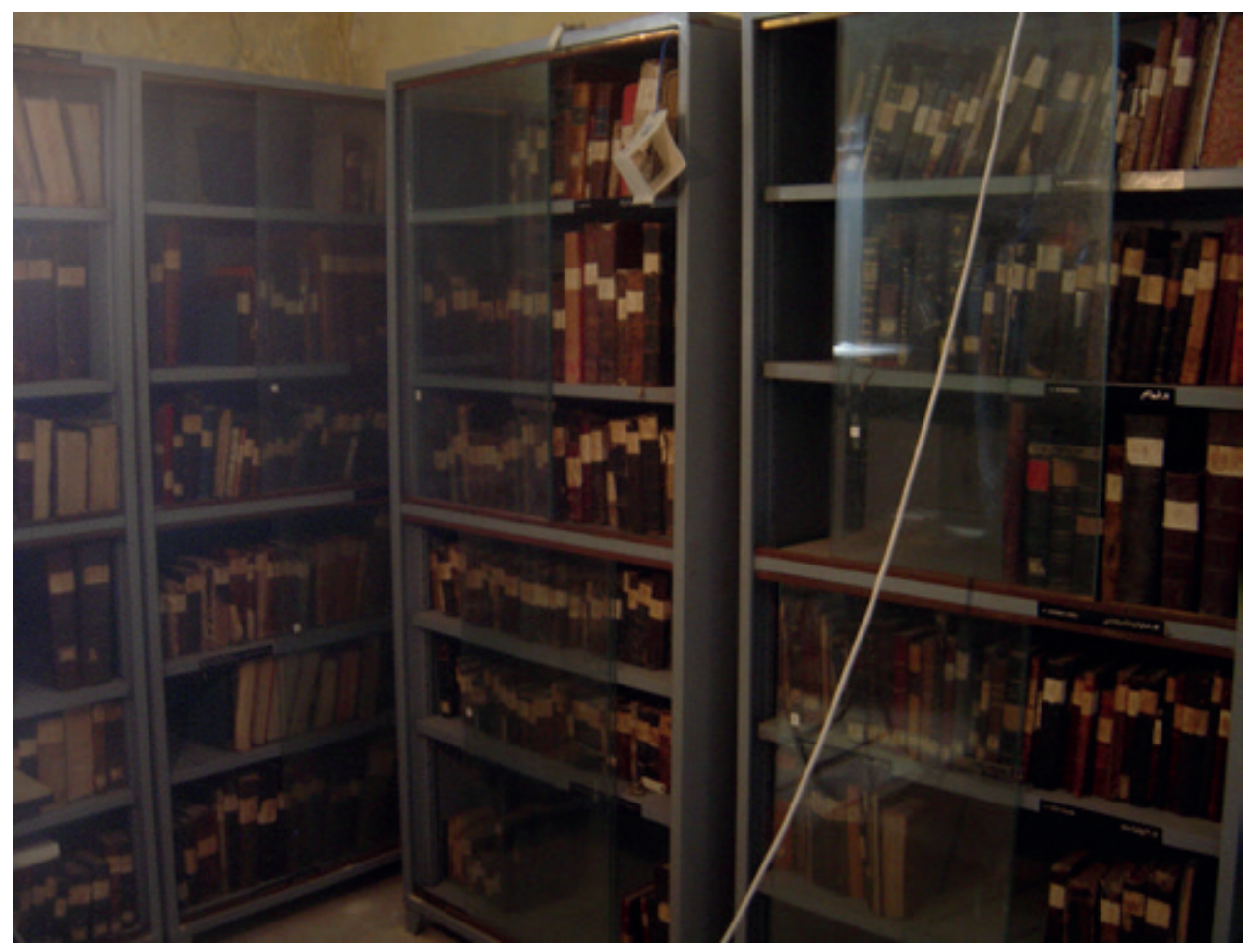

Monastère de Charfet, bibliothèque des manuscrits, fonds Charfet

Nisibe, mais la littérature plus récente et la tradition littéraire, liturgique et patristique sont différentes. L'écriture a évolué de façon différente et la langue présente des variantes, notamment dans la vocalisation, qui du coup a été notée, quand elle l'était, selon deux systèmes différents.

Les assemblages de manuscrits conservés dans les bibliothèques occidentales, européennes notamment, présentent des documents des différentes traditions, au hasard des achats et des dépôts qui y ont été faits. C'est notamment le cas à Paris où j'ai commencé mon travail sur le syriaque en établissant le catalogue de près d'une centaine de manuscrits. Travailler dans une bibliothèque du Proche-Orient, c'est au contraire se plonger dans une tradition particulière, ici la tradition syro-occidentale, celle de l'Église syro-orthodoxe, dite encore "monophysite " ou « jacobite », et de son Église-sœur syro-catholique. On a donc affaire à une collection à peu près homogène, même si quelques manuscrits syro-orientaux, melkites et maronites sont aussi conservés dans la collection. Les manuscrits vont, en général, du $15^{\mathrm{e}}$ au $19^{\mathrm{e}}$ siècles, jusqu'au début du $20^{\mathrm{e}}$.

Cataloguer des manuscrits, c'est d'abord identifier les textes qui y sont copiés. On y trouve tout ce qu'on attend dans une bibliothèque monastique et ecclésiastique. Une large majorité des manuscrits est liturgique : des rituels de baptême, de mariage et d'ordination, dont les pages de garde ont souvent servi de registres et qui proviennent de différentes paroisses. D'autres relèvent de la vie monastique : des exemplaires du Shhimo, le livre des Heures de l'Église syroorthodoxe pour les jours de la semaine et du Fenqito, qui contient les jours de fête; le Bet Gazo, recueil des hymnes classés selon les huit tons mélodiques ; des recueils d'anaphores. Le texte biblique est aussi souvent représenté par des livres à usage liturgique pour le service en commun ou la méditation personnelle : 


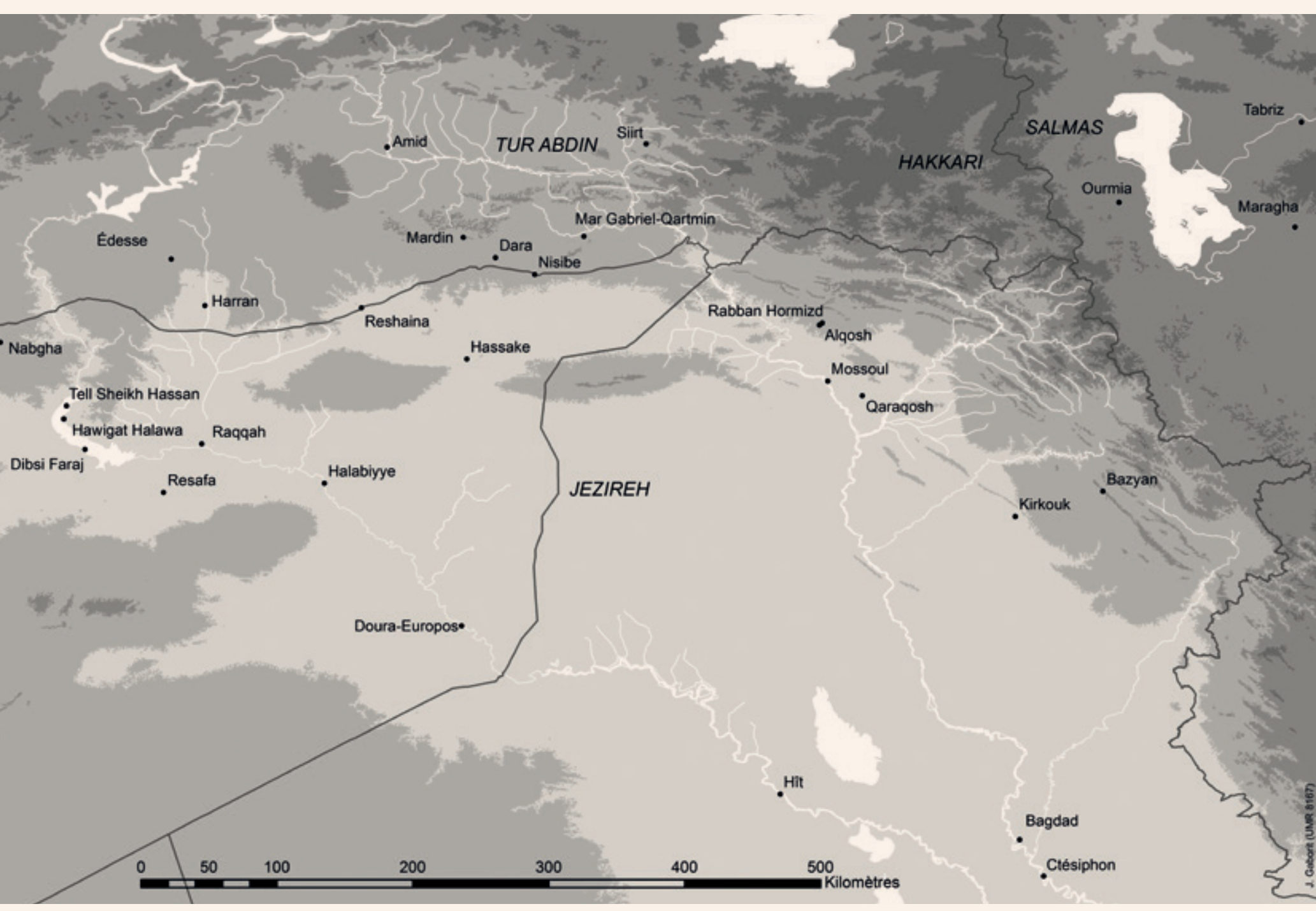

Carte de la Mésopotamie syriaque (Tur Abdin et région de Mossoul), réalisée par Justine Gaborit. 
psautiers et lectionnaires du Nouveau Testament ou de l'Ancien, mais aussi des Bibles organisées selon l'ordre des livres. Parmi les manuscrits destinés aux lectures spirituelles, on trouve des ouvrages apocryphes, dont l'Histoire de la Vierge Marie qui est particulièrement bien représentée, et des recueils d'hagiographie. Les autres manuscrits relèvent $d u$ travail intellectuel des moines : ouvrages d'exégèse et de commentaires bibliques, notamment de Denys bar Salibi, recueils d'hymnes et d'homélies métriques des grands auteurs syriaques, Éphrem, Jacques de Saroug, Isaac de Ninive ; ouvrages de spiritualité (particulièrement nombreux), de théologie, de patristique, de droit canonique, d'historiographie. Les traités de grammaire et ouvrages de lexicographie sont particulièrement bien représentés et montrent l'attachement de la communauté syriaque à sa propre langue, symbole de son identité chrétienne, dans un univers qui s'arabisait de plus en plus. De fait, les plus importants traités de lexicographie ont été composés à une époque où l'arabe gagnait du terrain.

Une attention particulière est portée dans notre travail aux colophons, souvent longs et très détaillés en syriaque, aux notes de copistes ou de possesseurs, qui sont systématiquement éditées. C'est une caractéristique des manuscrits syriaques d'avoir été systématiquement datés, et ce depuis le début du $5^{\mathrm{e}}$ siècle, alors que les premiers manuscrits datés, en grec comme en hébreu par exemple, remontent seulement au $9^{\mathrm{e}}$ siècle. L'usage de colophons développés a perduré jusque dans les manuscrits les plus récents. Ils permettent de voir l'origine de pièces qui n'ont qu'exceptionnellement été copiées au Mont Liban ou dans les environs, à Chypre par exemple. La plupart l'ont été dans deux régions : le nord de la Mésopotamie, autour de Mossoul et spécialement à Qaraqosh (Baghdeda), et le Tur Abdin dans le sud-est de la Turquie, dans les couvents de Mar Hanania, Deir al-Za'faran (couvent du safran) près de Mardin, ou de Mar Gabriel de Qartmin près de Midyat. Ils peuvent enfin provenir d'Amid (aujourd'hui Diyarbekir) sur le Tigre (voir carte ci-contre). Les lieux où ils ont été fabriqués, ceux où ils ont circulé et été conservés témoignent des réseaux d'échanges entre les monastères et les institutions, comme de l'histoire de l'Église syro-orthodoxe et, à partir de sa fondation, de sa sœur catholique. Ces deux régions correspondent en effet aux lieux où les chrétiens syro-orthodoxes se sont repliés à l'époque du déclin irrémédiable de leur Église, après les invasions mongoles de Tamerlan.

Pourquoi ces manuscrits sont-ils maintenant au Liban ? Leur arrivée est liée à l'histoire de la fondation de l'Église syro-catholique, qui a connu un processus compliqué. En 1656, un premier évêque syriaque d'Alep, André Akhijan, s'est rattaché à l'Église catholique. Il fut plus tard, en 1662, reconnu par la Sublime Porte, c'est-à-dire l'empire ottoman, comme patriarche catholique d'Antioche. Mais l'Église qu'il avait ainsi fondée était fragile, elle a fini par rester sans patriarche et par disparaître comme institution, même si le courant qu'elle représentait était toujours présent. Au $18^{\mathrm{e}}$ siècle, le métropolite Michel Jarweh, archevêque syriaque orthodoxe d'Alep, se convertit personnellement au catholicisme. En 1782, le Saint Synode de l'Église syriaque orthodoxe l'élut comme patriarche. Peu après son intronisation, il se déclara catholique. Il se fit reconnaitre comme patriarche de tous les syriaques et demanda à Rome la confirmation de sa charge. En 1783, l'Église syriaque catholique fut donc constituée par l'entrée en communion avec Rome d'une partie de l'Église syriaque orthodoxe. Cependant, la plus grande partie de la communauté syro-orthodoxe réagit et le synode élut un nouveau patriarche, qui fut aussitôt confirmé par la Sublime Porte. Face à ce changement inattendu, le patriarche Jarweh quitta précipitamment le Tur Abdin et s'enfuit à Bagdad, d'où il gagna la montagne libanaise où il s'installa en 1801, au nord de Beyrouth, dans le monastère de Charfet. La communauté syro-catholique, cependant, était restée dans les mêmes lieux et partageait les mêmes villages que les orthodoxes. Si la plupart des monastères sont restés orthodoxes, en Mésopotamie le couvent de Mar Behnam relève de la communauté catholique quand celui de Mar Mattaï est syro-orthodoxe. Le patrimoine écrit, et notamment manuscrit, est le même et c'est au hasard de rattachements individuels, de voyages personnels que certains manuscrits sont passés dans le patrimoine de l'Église syro-catholique. L'héritage est fondamentalement le même que celui de l'Église syroorthodoxe (voir ill. p. 39 en haut).

Un exemple spectaculaire l'illustrera : la découverte faite dans la reliure du manuscrit Charfet Rahmani 11 par deux membres de l'équipe, Youssef 
Dergham et Alain Desreumaux ${ }^{2}$. Il s'agit d'un recueil syro-orthodoxe de prières, notamment pour les malades. Il a été copié en 1790-1791 par le moine Rabban Hindi de Deir al-Za'faran pour Rabban 'Abd el-Ahad de Mardin. Pour fabriquer la reliure et notamment les ais, le relieur a coupé et collé les uns contre les autres des fragments d'un manuscrit très ancien en parchemin, selon une habitude bien connue. Le démontage de la reliure a permis de séparer et d'étudier ces feuillets, qui se sont révélés être des folios d'un manuscrit bien plus grand, pliés en deux. Le texte porté par les 8 folios ainsi récupérés est celui de la Bible syrohexaplaire, une traduction de la Bible faite en milieu syro-occidental au début du $7^{\mathrm{e}}$ siècle. Contrairement à l'ancienne version, la Peshitta, faite sur l'hébreu, qui est commune à toutes les Églises syriaques et qui est restée seule utilisée dans la liturgie, la syro-hexaplaire a été faite sur la version grecque des Septante, et porte en annotation les variantes des autres versions mises en colonnes dans les Hexaples d'Origène. C'est donc une version savante dont on n'a que très peu d'exemplaires. Or les caractéristiques codicologiques de ces folios, que l'on peut dater sans doute du $8^{\text {e }}$ siècle, ont permis de poser l'hypothèse qu'ils appartenaient à l'origine à un manuscrit du couvent syro-orthodoxe Saint-Marc à Jérusalem, qui contient ce texte et dont ils comblent partiellement une lacune. On peut reconstituer l'histoire de ce document ainsi : il devait être, à la fin du $18^{\mathrm{e}}$ ou au début du $19^{\mathrm{e}}$ siècle, dans le couvent de Mar Hanania à Mardin, déjà en mauvais état avec des folios qui se détachaient. Certains feuillets détachés et dissociés du manuscrit principal ont été réutilisés dans la reliure d'un nouveau manuscrit. Le reste de cette Bible syro-hexaplaire a été emporté, à une date que l'on ne peut préciser, au couvent SaintMarc de Jérusalem. Le nouveau manuscrit est quant à lui passé à l'Église syro-catholique et a rejoint le monastère de Charfet au Liban, avec la collection du patriarche Rahmani. Voilà donc une illustration de la circulation des documents entre les monastères, dont on a aussi maints exemples pour des périodes bien plus anciennes : si les colophons disent souvent pour quel monastère un manuscrit a été copié et recommandent de ne jamais l'en enlever, on voit que ces derniers sont souvent de grands nomades.
Les manuscrits de Charfet témoignent aussi du mélange des traditions scribales : l'écriture serțō, développée en milieu syro-occidental, a trouvé le chemin de la Mésopotamie où des communautés syro-orthodoxes se sont implantées. Dans la région de Mossoul, les copistes syro-orientaux ou syrooccidentaux utilisaient chacun leur écriture, bien caractéristique. Mais les syro-occidentaux ont subi l'influence de leurs collègues de la région et, à la place ou en même temps que leur propre système de vocalisation, par petites voyelles grecques sur- ou souscrites, ils ont aussi utilisé le système oriental des points-voyelles. Les caractéristiques codicologiques reflètent ainsi les échanges entre communautés, et notamment les influences réciproques avec l'Église syro-orientale de Mésopotamie (voir ill. ci-contre, en bas).

Un autre exemple de mélange de traditions est le système du "garshuni ", la coutume selon laquelle on écrit la langue arabe avec l'écriture syriaque $^{3}$. Ce système, qui a été surtout utilisé dans les communautés syro-occidentales, y compris maronites, est particulièrement bien représenté à Charfet. Il n'a pas d'utilité pratique, bien au contraire, puisque l'alphabet syriaque, qui comporte 22 lettres, est déficient pour noter les 28 phonèmes de la langue arabe. Développé particulièrement à partir des $15^{\mathrm{e}}$ et $16^{\mathrm{e}}$ siècles, à un moment où les communautés chrétiennes abandonnaient complètement leur langue d'origine pour l'arabe, il souligne la volonté de maintenir l'usage d'une écriture propre dans laquelle s'enracine une identité chrétienne.

\section{NOTES}

1- M. Debié, « Cataloguing in many ways: the case study of Charfet and e-ktobe ", dans Manuscripta syriaca. Des sources de première main, F. Briquel Chatonnet et M. Debié (éd.), Cahiers d'études syriaques, 4, Paris, Geuthner, 2015, p. 125-138.

2- Y. Dergham et A. Desreumaux, «Les surprises des reliures : découverte de fragments d'un ancien manuscrit syro-hexaplaire à Charfet ", dans Manuscripta syriaca. Des sources de première main, F. Briquel Chatonnet et M. Debié (éd.), Cahiers d'études syriaques, 4, Paris, Geuthner, 2015, p. 375-383.

3- F. Briquel Chatonnet, «Un cas d'allographie : le garshuni », dans Écriture et communication. Actes du colloque du CTHS, avril 2014, D. Briquel et F. Briquel Chatonnet (éd.), éditions électroniques du CTHS (Actes des congrès des sociétés historiques et scientifiques), Paris, 2015, p. 66-75 (http://cths.fr/ed/edition.php?id=6954). 


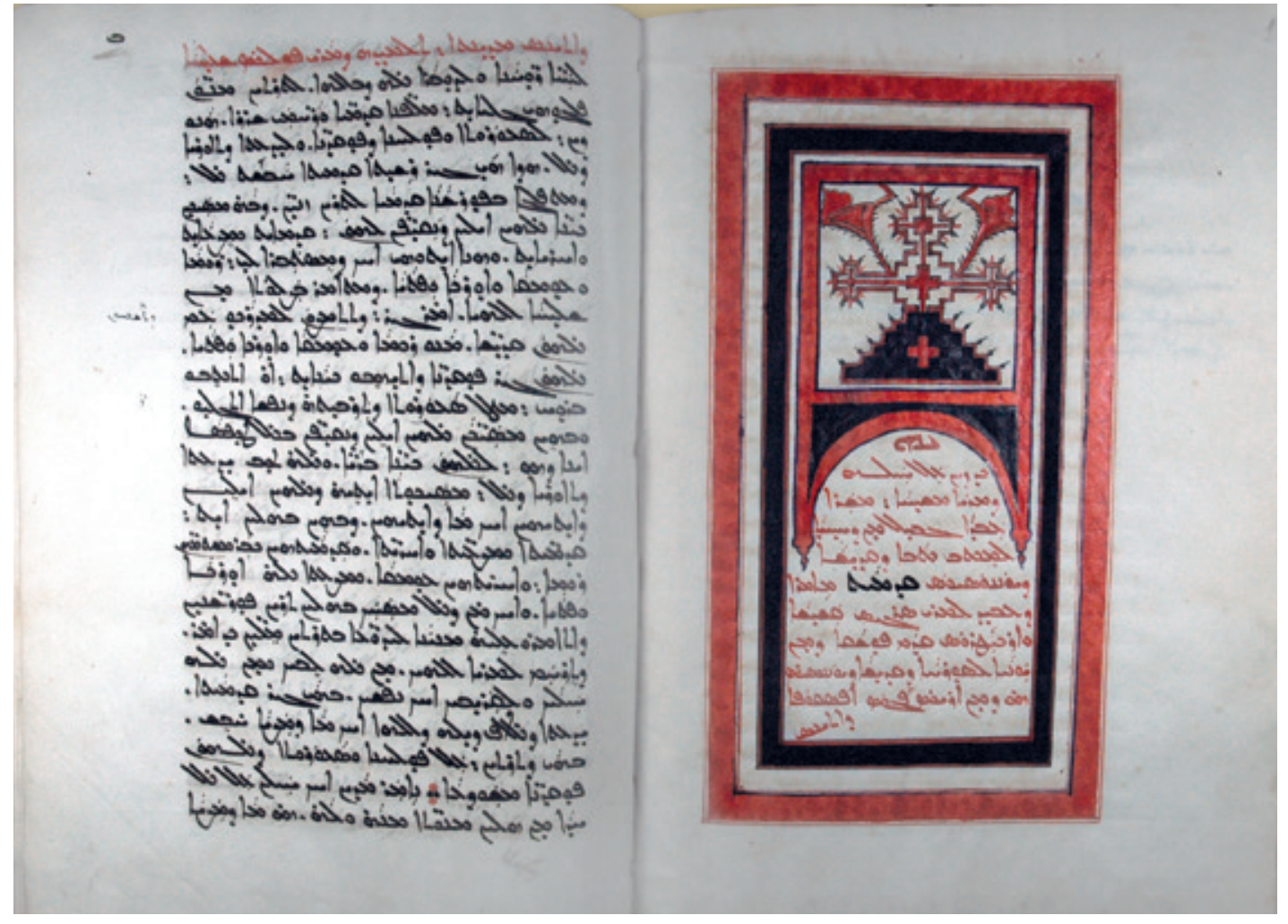

Ms. Charfet Rahmani 90, f. 1v-2r : Denys l'Aréopagite, Euvres traduites par Phocas avec ses scholia. Le texte commence par une préface de Serge de Reshaina (1903 de l'ère chrétienne ; coll. Bibliothèque de Charfet).

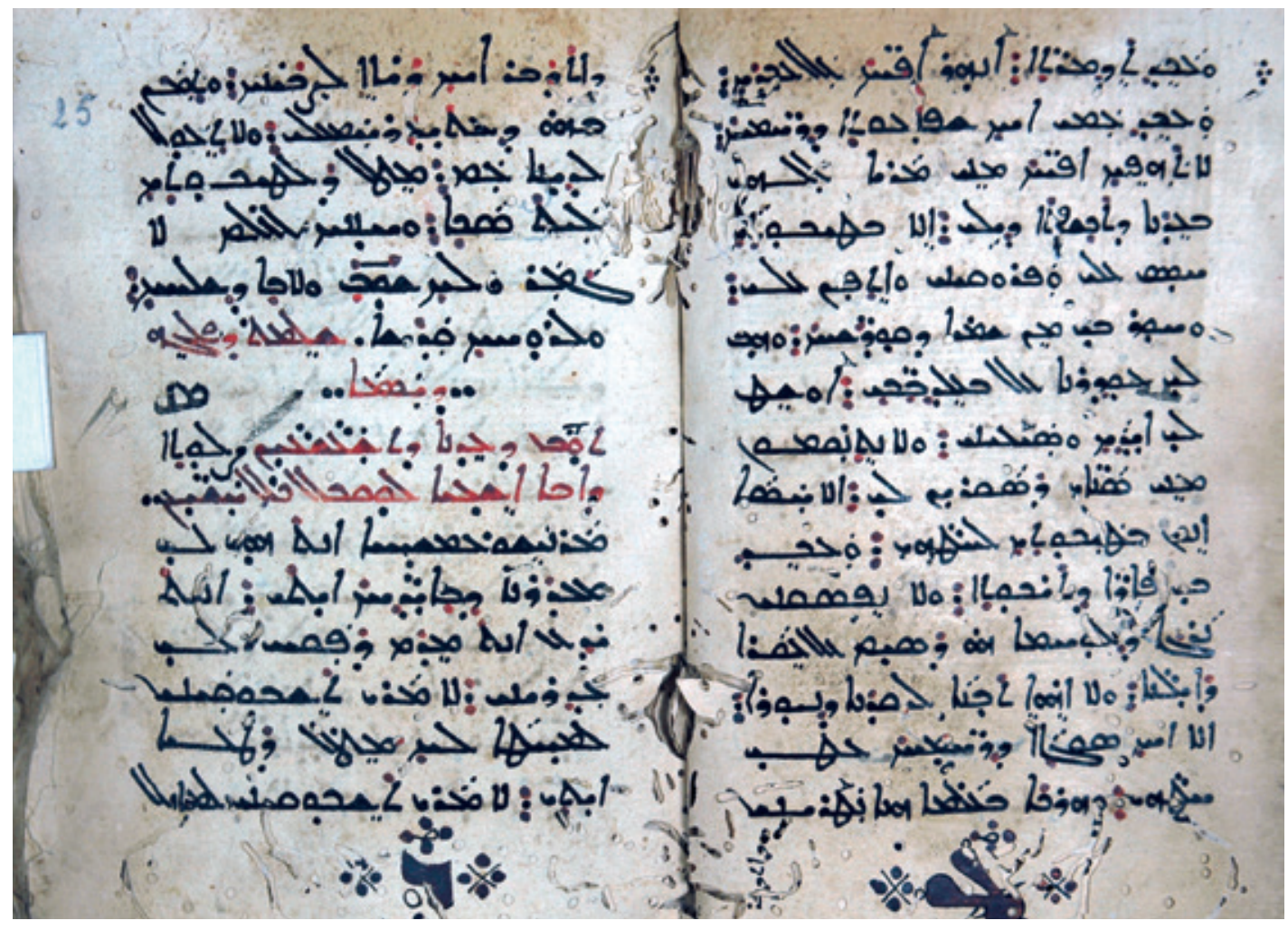

Ms. Charfet Rahmani 16, f. 24v-25r : Recueil de prières et de mimre du phanqito. $\mathrm{Au}$ f. $25 \mathrm{r}$, début d'une prière d'Abba Isaie (17 siècle ; coll. Bibliothèque de Charfet). 